\title{
ONE-SIDED CONGRUENCES ON INVERSE SEMIGROUPS
}

\author{
BY
}

\author{
JOHN MEAKIN
}

\begin{abstract}
By the kernel of a one-sided (left or right) congruence $\rho$ on an inverse semigroup $S$, we mean the set of $\rho$-classes which contain idempotents of $S$. We provide a set of independent axioms characterizing the kernel of a one-sided congruence on an inverse semigroup and show how to reconstruct the one-sided congruence from its kernel. Next we show how to characterize those partitions of the idempotents of an inverse semigroup $S$ which are induced by a one-sided congruence on $S$ and provide a characterization of the maximum and minimum one-sided congruences on $S$ inducing a given such partition. The final two sections are devoted to a study of indempotent-separating one-sided congruences and a characterization of all inverse semigroups with only trivial full inverse subsemigroups. A Green-Lagrange-type theorem for finite inverse semigroups is discussed in the fourth section.
\end{abstract}

1. Basic notions, terminology. We adhere throughout to the notation and terminology of A. H. Clifford and G. B. Preston [1]. Throughout the paper, $S$ will always denote an inverse semigroup (i.e., for each $a \in S$, there exists a unique element $a^{-1} \in S$ such that $a=a a^{-1} a$ and $a^{-1}=a^{-1} a a^{-1}$ ) and $E_{S}$ will denote the set of idempotents of $S$. The elementary properties of inverse semigroups may be found in [1]. In particular, we shall liberally use, without comment, the fact that $E_{S}$ is a semilattice (a commutative semigroup of idempotents) and that $a^{-1} E_{S} a \subseteq E_{S} \quad \forall a \in S$. We shall also use the fact that if $S$ is an inverse semigroup then the Green's relations $L$ and $R$ on $S$ are given by

$$
L=\left\{(a, b) \in S \times S: a^{-1} a=b^{-1} b\right\}
$$

and

$$
R=\left\{(a, b) \in S \times S: a a^{-1}=b b^{-1}\right\} .
$$

We denote the lattice of left congruences on $S$ by $L(S)$ and the lattice of right congruences on $S$ by $R(S)$. For each $\rho \in L(S) \cup R(S)$ we set

$$
\rho_{-1}=\left\{(a, b) \in S \times S:\left(a^{-1}, b^{-1}\right) \in \rho\right\} .
$$

It is obvious that $\rho_{-1}$ is a left (right) congruence on $S$ iff $\rho$ is a right (left) congruence on $S$, and that $\left(\rho_{-1}\right)_{-1}=\rho$.

Received by the editors May 29, 1973.

AMS (MOS) subject classifications (1970). Primary 20M10. 
Proposition 1.1. The lattice of left congruences on an inverse semigroup $S$ is isomorphic to the lattice of right congruences on $S$.

Proof. The mapping $\phi: \rho \rightarrow \rho_{-1} \forall \rho \in L(S)$ is easily seen to be a lattice isomorphism of $L(S)$ onto $R(S)$.

By virtue of this observation we feel at liberty to restrict our attention to left congruences on inverse semigroups throughout the remainder of the paper. Any result concerning left congruences on $S$ has an obvious dual result concerning right congruences on $S$.

We remark that it is clear that $R_{-1}=L$ and $L_{-1}=R$, where $R$ and $L$ denote the usual Green's relations on $S$ : note also that if $\rho \in R(S) \cup L(S)$, then $\rho_{-1}=\rho$ iff $\rho$ is a congruence on $S$. (This last remark follows from the fact that if $\rho$ is a congruence on an inverse semigroup then $(a, b) \in \rho$ iff $\left(a^{-1}, b^{-1}\right) \in \rho$; see, for example, J. M. Howie [3] .)

2. The kernel of a left congruence on an inverse semigroup. If $\rho$ is a left congruence on an inverse semigroup $S$ then the kernel of $\rho$ is the set of $\rho$-classes which contain indempotents of $S$, i.e.,

$$
\operatorname{Ker} \rho=\left\{e \rho: e \in E_{S}\right\} \text {. }
$$

It is well known that if $\rho$ and $\sigma$ are two left congruences on an inverse semigroup, then $\rho=\sigma$ iff $\operatorname{Ker} \rho=\operatorname{Ker} \sigma$. (See [1, Theorem 7.39].) This result naturally suggests the following question: How do we characterize the kernel of a left congruence on an inverse semigroup and how do we reconstruct the left congruence from its kernel? In Theorem 2.1 we provide an answer to this question. The corresponding problem for two-sided congruences on inverse semigroups has been solved by G. B. Preston [4].

We introduce the following notation. If $\mathrm{A}=\left\{A_{i}: i \in I\right\}$ is a nonempty set of disjoint subsets of $S$ then we shall consistently use the notation $A(\mathrm{~A})=$ $\bigcup_{i \in I} A_{i}$ (or more simply $A(\mathrm{~A})=A$ if no confusion can arise) and if $a \in A$, then $A(a)$ will denote the element $A_{i}$ of $\mathrm{A}$ to which $a$ belongs, i.e. $A_{i}=$ $A(a)$ iff $a \in A_{i}$.

Definition 2.1. A set $A=\left\{A_{i}: i \in I\right\}$ of disjoint subsets of the inverse semigroup $S$ is called a left kernel system of $S$ if it satisfies the conditions:

(L1) $E_{S} \subseteq A$;

(L2) for each $i \in I, E_{S} \cap A_{i} \neq \square$;

(L3) for each $a \in A$ and $j \in I, \exists k \in I$ such that $a A_{j} \subseteq A_{k}$;

(L4) for each $a \in A, a^{-1} A(a) \subseteq A\left(a^{-1} a\right)$;

(L5) if $a^{-1} b \in A\left(a^{-1} a\right)$ for some $b \in A$, then $a \in A$.

(We remark that L3 implies in particular that $A$ is a subsemigroup of $S$.) 
Lemma 2.1. If $\rho$ is a left congruence on $S$ with $\operatorname{Ker} \rho=\left\{A_{i}: i \in I\right\}$, then $\operatorname{Ker} \rho$ is a left kernel system of $S$.

Proof. Ker $\rho$ clearly satisfies $\mathrm{L} 1, \mathrm{~L} 2$ and $\mathrm{L} 4$. Let $a \in A, j \in I$ and $f \in A_{j} \cap E_{S}, e \in A(a) \cap E_{S}$. Then for each $b \in A_{j}, a b \rho a f=a a^{-1} a f=$ $\left(a f a^{-1}\right) a \rho\left(a f a^{-1}\right) e \in E_{S}$.

Thus $a A_{j} \subseteq A\left(\left(a f a^{-1}\right) e\right)$, and so ker $\rho$ satisfies L3. Finally, if $a^{-1} b \in$ $A\left(a^{-1} a\right)$ for some $b \in A$ and if $g \in A(b) \cap E_{S}$, then

$$
a=a\left(a^{-1} a\right) \rho a\left(a^{-1} b\right)=\left(a a^{-1}\right) b \rho\left(a a^{-1}\right) g \in E_{S},
$$

and so $a \in A$.

We make two small remarks about Lemma 2.1 and Definition 2.1. The lemma implies that even though each element $e \rho$ of $\operatorname{Ker} \rho$ is not necessarily a subsemigroup of $S$ (for example an $R$-class of $S$ is not necessarily a subsemigroup of $S$ but $R$ is a left congruence on $S$ ), the union $U_{e \in E_{S}} e \rho$ of the elements of $\operatorname{Ker} \rho$ is always a subsemigroup of $S$. We also remark that, in the special case in which $S$ is a group with identity $e, \operatorname{Ker} \rho=\{A(e)\}$ and conditions $\mathrm{L} 1-\mathrm{L} 5$ reduce simply to the condition that $A(e)$ is a subgroup of $S$.

The next theorem tells us that conditions L1-L5 provide the desired characterization of the kernel of a left congruence on an inverse semigroup.

TheOREM 2.1. If $\mathrm{A}=\left\{A_{i}: i \in I\right\}$ is a left kernel system of $S$ then the relation

$$
\rho_{\mathrm{A}}=\left\{(a, b) \in S \times S: a^{-1} b \in A\left(a^{-1} a\right) \text { and } b^{-1} a \in A\left(b^{-1} b\right)\right\}
$$

is a left congruence on $S$ with kernel $A$. If $\rho$ is a left congruence on $S$ with kernel A then $A$ is a left kernel system of $S$ and $\rho=\rho_{A}$.

Proof. In view of Lemma 2.1 and the uniqueness theorem of Clifford and Preston it suffices to show that if $A$ is a left kernel system of $S$ then $\rho_{A}$ is a left congruence on $S$ with kernel A. Let A satisfy L1-L5 and introduce the following notation: $a \sim b$ iff $a$ and $b$ are in the same element $A_{i}$ of $A$, i.e., $a \sim b$ iff $a, b \in A_{i}$ for some $i \in I$. Note that $\sim$ is a partial equivalence relation on $S$.

It is clear that $\rho_{A}$ is reflexive and symmetric: To prove that $\rho_{A}$ is transitive let $(a, b) \in \rho_{\mathrm{A}}$ and $(b, c) \in \rho_{\mathrm{A}}$. Then $a^{-1} b \sim a^{-1} a, b^{-1} a \sim b^{-1} b$, $b^{-1} c \sim b^{-1} b$ and $c^{-1} b \sim c^{-1} c$. Hence, by L3 and L1,

$$
c^{-1} a a^{-1} c=\left(c^{-1} a a^{-1} c\right) c^{-1} c \sim\left(c^{-1} a a^{-1} c\right) c^{-1} b=c^{-1} a a^{-1} b .
$$

Hence $\left(a^{-1} c\right)^{-1}\left(a^{-1} b\right) \in A\left(\left(a^{-1} c\right)^{-1}\left(a^{-1} c\right)\right)$ and $a^{-1} b \in A$ and it follows by L5 that $a^{-1} c \in A$. Thus by many applications of L3 we have 


$$
\begin{aligned}
a^{-1} c & =a^{-1} c c^{-1} c \sim a^{-1} c c^{-1} b=a^{-1} c c^{-1} b b^{-1} b \sim a^{-1} c c^{-1} b b^{-1} a \\
& =a^{-1} c c^{-1} b b^{-1} a a^{-1} a \sim a^{-1} c c^{-1} b b^{-1} a a^{-1} b \\
& =a^{-1} b b^{-1} c c^{-1} b \sim a^{-1} b b^{-1} c c^{-1} c=a^{-1} b b^{-1} c \sim a^{-1} b b^{-1} b \\
& =a^{-1} b \sim a^{-1} a .
\end{aligned}
$$

Hence $a^{-1} c \in A\left(a^{-1} a\right)$, and a similar argument shows that $c^{-1} a \in A\left(c^{-1} c\right)$. Thus $(a, c) \in \rho_{\mathrm{A}}$ and so $\rho_{\mathrm{A}}$ is transitive.

Now suppose that $(a, b) \in \rho_{A}$ and $c \in S$. Then $a^{-1} b \sim a^{-1} a, b^{-1} a \sim$ $b^{-1} b$, and, by $\mathrm{L} 3$,

$$
\begin{aligned}
(c a)^{-1} c a & =a^{-1} c^{-1} c a=\left(a^{-1} c^{-1} c a\right) a^{-1} a \sim a^{-1} c^{-1} c a a^{-1} b \\
& =a^{-1} c^{-1} c b=(c a)^{-1} c b,
\end{aligned}
$$

and similarly $(c b)^{-1} c b \sim(c b)^{-1} c a$. It follows that $\rho_{\mathrm{A}}$ is a left congruence on $S$.

Now let $K=\left\{K_{j}: j \in J\right\}$ be the kernel of $\rho_{A}$. We let $K_{j} \in K$ and show first that $K_{j} \subseteq A_{i}$ for some $i \in I$. Choose $e \in K_{j} \cap E_{S}$ and let $k \in K_{j}$. Then $(k, e) \in \rho_{\mathrm{A}}$ and so $k^{-1} k \sim k^{-1} e$ and $e k \sim e$. Since $k^{-1} k \sim k^{-1} e$ it follows that $k \in A$ by L5. Hence, by L3, $k=k k^{-1} k \sim k k^{-1} e \sim k k^{-1}(e k)=e k \sim e$, so $k \in A(e)$. It follows that $K_{j} \subseteq A(e)$.

Conversely, let $A_{i} \in \mathrm{A}$ and suppose that $e \in A_{i} \cap E_{S}$. Then $e \in K_{j}$ for some $j \in J$. Let $a \in A_{i}$. Then $a \sim e$ and so $e a \sim e$ by L3. Also, $a^{-1} e \in$ $a^{-1} A(a) \subseteq A\left(a^{-1} a\right)$ by L4. It follows that $a^{-1} e \sim a^{-1} a$ and this, together with $e a \sim e$, implies that $(a, e) \in \rho_{\mathrm{A}}$, i.e., $a \in K_{j}$, and so $A_{i} \subseteq K_{j}$. Thus $K=\mathrm{A}$ is the kernel of $\rho_{A}$. This completes the proof of the theorem.

We now provide examples which show that the conditions L1-L5 are mutually independent. Let $I_{2}$ be the symmetric inverse semigroup on two letters $\{1,2\}$. ( $I_{2}$ is the set of all one-one mappings from subsets of $\{1,2\}$ onto subsets of $\{1,2\}$ (including the empty mapping) with composition defined in the usual way-see [1] for details.) We use the notation $\left(\begin{array}{ll}x & y \\ z & w\end{array}\right)$ for the mapping which takes $x$ to $z$ and $y$ to $w,\left(\begin{array}{l}x \\ y\end{array}\right)$ for the mapping which takes $x$ to $y$, and 0 for the empty mapping. Note that

$$
E_{I_{2}}=\left\{\left(\begin{array}{ll}
1 & 2 \\
1 & 2
\end{array}\right),\left(\begin{array}{l}
1 \\
1
\end{array}\right),\left(\begin{array}{l}
2 \\
2
\end{array}\right), 0\right\} .
$$

EXAMPLE 1. Let

$$
A=\left\{\left\{\left(\begin{array}{ll}
1 & 2 \\
1 & 2
\end{array}\right),\left(\begin{array}{l}
1 \\
1
\end{array}\right)\right\},\left\{0,\left(\begin{array}{l}
2 \\
2
\end{array}\right)\right\}\right\} .
$$

One may readily check that $A$ satisfies conditions L1-L4 but not L5. 
EXAMPLE 2. Let

$$
A=\left\{\left\{\left(\begin{array}{ll}
1 & 2 \\
1 & 2
\end{array}\right)\right\},\left\{\left(\begin{array}{l}
1 \\
1
\end{array}\right)\right\},\left\{\left(\begin{array}{l}
2 \\
2
\end{array}\right)\right\},\left\{0,\left(\begin{array}{l}
1 \\
2
\end{array}\right)\right\}\right\} .
$$

Conditions $\mathrm{L} 1, \mathrm{~L} 2, \mathrm{~L} 3$, and $\mathrm{L} 5$ are satisfied but $\mathrm{L} 4$ is not.

EXAMPLE 3. If

$$
A=\left\{\left\{\left(\begin{array}{l}
1 \\
2
\end{array}\right),\left(\begin{array}{l}
1 \\
1
\end{array}\right)\right\},\left\{\left(\begin{array}{l}
2 \\
1
\end{array}\right),\left(\begin{array}{l}
2 \\
2
\end{array}\right)\right\},\left\{\left(\begin{array}{ll}
1 & 2 \\
1 & 2
\end{array}\right), 0,\left(\begin{array}{ll}
1 & 2 \\
2 & 1
\end{array}\right)\right\}\right\},
$$

then A satisfies L1, L2, L4 and L5 but not L3.

Example 4. Let $A=\{\{0\}\}, A$ satisfies L2-L5 but not $L 1$.

EXAMPLE 5. If

$$
A=\left\{\{0\},\left\{\left(\begin{array}{l}
1 \\
1
\end{array}\right)\right\},\left\{\left(\begin{array}{l}
2 \\
2
\end{array}\right)\right\},\left\{\left(\begin{array}{l}
1 \\
2
\end{array}\right)\right\},\left\{\left(\begin{array}{l}
2 \\
1
\end{array}\right)\right\},\left\{\left(\begin{array}{ll}
1 & 2 \\
1 & 2
\end{array}\right)\right\}\right\},
$$

then conditions L1, L3, L4 and L5 are satisfied but L2 is not satisfied.

Thus no four of L1-L5 imply the fifth and so L1-L5 are mutually independent. We make the remark that while it is true that if $\rho$ is a left congruence on $S$ then $A=\bigcup_{e \in E_{S}}^{e \rho}$ is a subsemigroup of $S$, it is not true that $A$ is necessarily an inverse subsemigroup. For example the partition

$$
\left\{\left(\begin{array}{ll}
1 & 2 \\
1 & 2
\end{array}\right),\left(\begin{array}{l}
1 \\
1
\end{array}\right)\right\},\left\{\left(\begin{array}{ll}
1 & 2 \\
2 & 1
\end{array}\right),\left(\begin{array}{l}
2 \\
1
\end{array}\right)\right\},\left\{\left(\begin{array}{l}
1 \\
2
\end{array}\right), 0,\left(\begin{array}{l}
2 \\
2
\end{array}\right)\right\}
$$

defines a left congruence on $I_{2}$ and here

$$
A=\left\{\left(\begin{array}{ll}
1 & 2 \\
1 & 2
\end{array}\right),\left(\begin{array}{l}
1 \\
1
\end{array}\right),\left(\begin{array}{l}
1 \\
2
\end{array}\right), 0,\left(\begin{array}{l}
2 \\
2
\end{array}\right)\right\}
$$

is not an inverse subsemigroup of $I_{2}$.

3. Idempotentequivalent one-sided congruences. The set of left congruences on $S$ which induce the same fixed partition $E=\left\{E_{i}: i \in I\right\}$ of $E_{S}$ constitutes a complete sublattice of $L(S)$ (with both 0 and 1 elements). Two left congruences on $S$ are called idempotent-equivalent if they induce the same partition of $E_{S}$. In view of these remarks it is natural to ask which partitions of $E_{S}$ are induced by left congruences on $S$ and to seek a characterization of the maximum and minimum left congruences on $S$ which induce a given partition of $E_{S}$. We provide the solution to this problem in the following theorem. Reilly and Scheiblich have answered the corresponding set of questions for two-sided congruences on inverse semigroups in [5].

THEOREM 3.1. A partition $E=\left\{E_{i}: i \in I\right\}$ of $E_{S}$ into disjoint subsets is induced by some left congruence on $S$ iff $E$ satisfies:

(N) $\forall i, j \in I, \quad \exists k=k(i, j) \in I$ such that $E_{i} E_{j} \subseteq E_{k}$.

The minimum left congruence on $S$ inducing such a partition of $E_{S}$ is 
$\xi=\left\{(a, b) \in S \times S: \exists i \in I\right.$ such that $a^{-1} a, b^{-1} b \in E_{i}$ and

$$
a e=b e \text { for some } e \in E_{i} \text { \}. }
$$

The maximum left congruence on $S$ inducing such a partition of $E_{S}$ is

$$
\begin{aligned}
& \zeta=\left\{(a, b) \in S \times S: a^{-1} b b^{-1} a \in E\left(a^{-1} a\right), b^{-1} a a^{-1} b \in E\left(b^{-1} b\right)\right. \text { and for } \\
& \text { each } k \in I, \exists l, m \in I \text { such that } a^{-1} b E_{k} b^{-1} a \subseteq E_{l} \text { and } \\
& \left.b^{-1} a E_{k} a^{-1} b \subseteq E_{m}\right\} .
\end{aligned}
$$

Proof. Let $\rho$ be a left congruence on $S$ and let $E=\left\{E_{i}: i \in I\right\}$ be the partition of $E_{S}$ induced by $\rho$. It is an easy matter to see that $E$ satisfies the condition (N), for if $e_{1}, e_{2} \in E_{i}$ and $f_{1}, f_{2} \in E_{j}$ it follows that $e_{1} f_{1} \rho e_{1} f_{2}=$ $f_{2} e_{1} \rho f_{2} e_{2}=e_{2} f_{2}$ and so $E_{i} E_{j} \subseteq E_{k}$ for some $k \in I$.

Suppose now that $E$ is a partition of $E_{S}$ which satisfies (N). We show that there is some left congruence on $S$ which induces this partition $E$ of $E_{S}$ by showing directly that both $\xi$ and $\zeta$ have this property. We first prove that $\xi$ is a left congruence on $S$ with the properties stated in the theorem.

Evidently, $\xi$ is reflexive and symmetric. Suppose now that $(a, b) \in \xi$ and $(b, c) \in \xi$ : Then there exists $i \in I$ such that $a^{-1} a, b^{-1} b, c^{-1} c \in E_{i}$ and $a e=$ $b e, b f=c f$ for some $e, f \in E_{i}$. Then $a(e f)=b e f=b f e=c f e=c(e f)$, and $e f \in E_{i}$, so $(a, c) \in \xi$. Now let $c$ be any element of $S$ and suppose that $a^{-1} c^{-1} c a \in E_{j}$. Then $\left(a^{-1} a\right)\left(a^{-1} c^{-1} c a\right)\left(a^{-1} a\right) \in E_{j}$ and it follows by $(\mathrm{N})$ that

$$
e\left(b^{-1} c^{-1} c b\right) e=\left(e b^{-1}\right)\left(c^{-1} c\right)(b e)=e\left(a^{-1} c^{-1} c a\right) e \in E_{j},
$$

and hence that $b^{-1} c^{-1} c b=\left(b^{-1} b\right)\left(b^{-1} c^{-1} c b\right)\left(b^{-1} b\right) \in E_{j}$. Furthermore, if we let $f=e a^{-1} c^{-1} c a=e a^{-1} c^{-1} c a e=e b^{-1} c^{-1} c b e=b^{-1} c^{-1} c b e$, we easily see that $f \in E_{j}$ and that

$$
c a f=c a a^{-1} c^{-1} c a e=c a e=c b e=c b b^{-1} c^{-1} c b e=c b f,
$$

and hence $(c a, c b) \in \xi$. Thus $\xi$ is a left congruence on $S$.

It is almost immediate from the definition of $\xi$ that if $e, f \in E_{S}$, then $(e, f) \in \xi$ iff $e, f \in E_{i}$ for some $i \in I$. Thus $\xi$ induces the partition $E$ of $E_{S}$ and it remains to show that if $\rho$ is any left congruence on $S$ which induces this partition $E$ of $E_{S}$, then $\xi \subseteq \rho$. Let $(a, b) \in \xi$ : There exists $i \in I$ such that $a^{-1} a, b^{-1} b \in E_{i}$ and $a e=b e$ for some $e \in E_{i}$. Since $\rho$ is a left congruence on $S$ which induces the partition $E$ of $E_{S}$ it follows that $a E_{i} \subseteq$ $a(e \rho) \subseteq x \rho$ for some $x \in S$. But $a=a\left(a^{-1} a\right) \in a E_{i}$ and so $a \in x \rho$. By a similar arguement, $b E_{i} \subseteq y \rho$ and $b \in y \rho$ for some $y \in S$, but since $a e=$ $b e \in a E_{i} \cap b E_{i}$, it follows that $x \rho=y \rho$ and that $a, b \in x \rho$. Thus $(a, b) \in \rho$ and so $\xi \subseteq \rho$. This completes the proof of the claims made about $\xi$. 
Now let $\zeta$ be the relation defined in the statement of the theorem. That $\zeta$ is reflexive follows from $(\mathrm{N})$, and that $\zeta$ is symmetric is immediately obvious. Suppose now that $(a, b) \in \zeta$ and $(b, c) \in \zeta$. Then there are elements $i, j, k \in I$ such that $a^{-1} a, a^{-1} b b^{-1} a \in E_{i}, b^{-1} b, b^{-1} a a^{-1} b, b^{-1} c c^{-1} b \in E_{j}, c^{-1} c$, $c^{-1} b b^{-1} c \in E_{k}$, and for each $l \in I$, there exist $m, n, p, q \in I$ such that $a^{-1} b E_{l} b^{-1} a \subseteq E_{m}, \quad b^{-1} a E_{l} a^{-1} b \subseteq E_{n}, \quad b^{-1} c E_{l} c^{-1} b \subseteq E_{p}$ and $c^{-1} b E_{l} b^{-1} c \subseteq$ $E_{q}$. To prove the transitivity of $\zeta$, we need to prove that $a^{-1} c c^{-1} a \in E_{i}$ and $c^{-1} a a^{-1} c \in E_{k}$, and that if $l \in I$, there exist $r, s \in I$ such that $a^{-1} c E_{t} c^{-1} a \subseteq$ $E_{r}$ and $c^{-1} a E_{l} a^{-1} c \subseteq E_{S}$. Now $a^{-1} c c^{-1} a=\left(a^{-1} c c^{-1} a\right)\left(a^{-1} a\right)$, so by $(\mathrm{N})$, $a^{-1} c c^{-1} a$ is in the same element of $E$ as.$\left(a^{-1} c c^{-1} a\right)\left(a^{-1} b b^{-1} a\right)$. But

$$
\left(a^{-1} c c^{-1} a\right)\left(a^{-1} b b^{-1} a\right)=a^{-1} b\left(b^{-1} c c^{-1} b\right) b^{-1} a \in a^{-1} b E_{j} b^{-1} a,
$$

and $a^{-1} b b^{-1} a=a^{-1} b\left(b^{-1} b\right) b^{-1} a \in a^{-1} b E_{j} b^{-1} a$. Hence $a^{-1} b E_{j} b^{-1} a \cap E_{i}$ $\neq \square$, and it follows that $a^{-1} b E_{j} b^{-1} a \subseteq E_{i}$. Hence $a^{-1} c c^{-1} a \in E_{i}$ and similarly $c^{-1} a a^{-1} c \in E_{k}$. Now choose $l \in I$ arbitrarily and let $e \in E_{l}$. Then, by $(\mathrm{N})$, $a^{-1} \operatorname{cec}^{-1} a$ is in the same element of $E$ as $\left(a^{-1} \operatorname{cec}^{-1} a\right)\left(a^{-1} b b^{-1} a\right)$, since $a^{-1} \operatorname{cec}^{-1} a=\left(a^{-1} \operatorname{cec}^{-1} a\right)\left(a^{-1} a\right)$. But

$$
\left(a^{-1} c e c^{-1} a\right)\left(a^{-1} b b^{-1} a\right)=a^{-1} b b^{-1} c e c^{-1} b b^{-1} a \in a^{-1} b\left(b^{-1} c E_{l} c^{-1} b\right) b^{-1} a,
$$

and $a^{-1} b\left(b^{-1} c E_{l} c^{-1} b\right) b^{-1} a \subseteq E_{r}$ for some $r \in I$, since there exist $p, r \in I$ for which $b^{-1} c E_{l} c^{-1} b \subseteq E_{p}$ and $a^{-1} b E_{p} b^{-1} a \subseteq E_{r}$. Hence $a^{-1} c e c^{-1} a \in E_{r}$ for each $e \in E_{l}$ and it follows that $a^{-1} c E_{l} c^{-1} a \subseteq E_{r}$. Similarly, $c^{-1} a E_{a^{-1}} c$ $\subseteq E_{s}$, some $s \in I$, and so $\zeta$ is an equivalence relation on $S$. Now let $c$ be any element of $S$ and let $(a, b) \in \zeta$ as above, and suppose that $a^{-1} c^{-1} c a \in E_{p}$. Then since $a^{-1} c^{-1} c a=\left(a^{-1} c^{-1} c a\right)\left(a^{-1} a\right)$, we have that $E_{p} E_{i} \subseteq E_{p}$. It follows that $a^{-1} c^{-1} c b$. $b^{-1} c^{-1} c a=\left(a^{-1} c^{-1} c a\right)\left(a^{-1} b b^{-1} a\right) \in E_{p}$, and the proof that $b^{-1} c^{-1} c b$ and $b^{-1} c^{-1} c a$. $a^{-1} c^{-1} c b$ are in the same element of $E$ is similar. Further, if $l \in I$ and $e \in E_{l}$, then

$$
\begin{aligned}
a^{-1} c^{-1} c b e b^{-1} c^{-1} c a & =\left(a^{-1} c^{-1} c a\right)\left(a^{-1} b e b^{-1} a\right) \in\left(a^{-1} c^{-1} c a\right)\left(a^{-1} b E_{l} b^{-1} a\right) \\
& \subseteq E_{p} E_{m} \subseteq E_{q}, \text { for some } q \in I,
\end{aligned}
$$

and it follows that $a^{-1} c^{-1} c b E_{l} b^{-1} c^{-1} c a \subseteq E_{q}$. Similarly $b^{-1} c^{-1} c a E_{l} a^{-1} c^{-1} c b$ $\subseteq E_{r}$ for some $r \in I$, and so $\zeta$ is a left congruence on $S$.

Again, it follows fairly easily that $\zeta$ induces the partition $E$ of $E_{S}$ : Let $\rho$ be any left congruence on $S$ which induces the partition $E$ of $E_{S}$, and let $(a, b) \in$ $\rho$. Then $\left(a^{-1} a, a^{-1} b\right) \in \rho$ and $\left(b^{-1} a, b^{-1} b\right) \in \rho$ and also $\left(b^{-1} a, b^{-1} a a^{-1} b\right)=$ $\left(b^{-1} a a^{-1} a, b^{-1} a a^{-1} b\right) \in \rho$. Since $\left(b^{-1} a, b^{-1} b\right) \in \rho$ it follows that $\left(b^{-1} b\right.$, $\left.b^{-1} a a^{-1} b\right) \in \rho$ and hence that $b^{-1} b, b^{-1} a a^{-1} b \in E_{i}$ for some $i \in I$. Similarly $a^{-1} a, a^{-1} b b^{-1} a \in E_{j}$ for some $j \in I$. Now let $l \in I$ and $e \in E_{l}$. Then 
$\left(e a^{-1} b, e a^{-1} a\right) \in \rho$, i.e., $\left(e a^{-1} b, a^{-1} a e\right) \in \rho$, and hence $\left(b^{-1} a e a^{-1} b, b^{-1} a a^{-1} a e\right)$ $\in \rho$, i.e., $\left(b^{-1} a e a^{-1} b, b^{-1} a e\right) \in \rho$. Let $A=\left\{A_{i}: i \in I\right\}$ be the kernel of $\rho$. Then $b^{-1} a e \in b^{-1} a E_{l} \subseteq b^{-1} a A_{l}$, and since $b^{-1} a e a^{-1} b \in A_{j}$ for some $j \in I$, we have $b^{-1} a A_{l} \subseteq A_{j}$ by the left congruence property of $\rho$. In particular, $b^{-1} a f \in A_{j}$ for all $f \in E_{l}$. Hence $b^{-1} a f a^{-1} b \in A_{j}$ for all $f \in E_{l}$, and it follows that $b^{-1} a E_{l} a^{-1} b \subseteq A_{j}$. Since $b^{-1} a E_{l} a^{-1} b \subseteq E_{S}$, we evidently have that $b^{-1} a E_{l} a^{-1} b \subseteq E_{j}$. Similarly, $a^{-1} b E_{l} b^{-1} a \subseteq E_{k}$, for some $k \in I$. Hence $(a, b)$ $\in \zeta$ and so $\rho \subseteq \zeta$. This completes the proof of the theorem.

We mention two corollaries of the theorem which are of independent interest and may be proved directly.

Corollary 3.1. A partition $E=\left\{E_{i}: i \in I\right\}$ of $E_{S}$ is induced by a left congruence on $S$ iff $E$ is induced by a right congruence on $S$.

(This follows from the left-right symmetry of condition $(\mathrm{N})$.)

Corollary 3.2. Any left (right, two-sided) congruence on $E_{S}$ may be extended to a left or right congruence on $S$ whose restriction to $E_{S}$ coincides with the original left congruence on $E_{S}$.

(This follows since condition (N) is of course just the condition which insures that the partition $E$ defines a congruence on the semilattice $E_{S}$.)

4. Idempotent-separating one-sided congruences. A one-sided congruence $\rho$ on $S$ is called idempotent-separating if distinct idempotents of $S$ are in distinct equivalence classes of $\rho$. Idempotent-separating left congruences on $S$ are clearly idempotent-equivalent in the sense of the previous section. For idempotentseparating left congruences on $S$ we are able to obtain a Green-Lagrange type theorem (Theorem 4.3): We first show how the results of the previous two sections may be simplified in the idempotent-separating case.

THEOREM 4.1. The maximum idempotent-separating left (right) congruence on $S$ is $R(L)$.

Proof. This may be proved by noting that the relation $\zeta$ of Theorem 3.1 reduces to $R$ in the special case in which $E=\left\{\{e\}: e \in E_{S}\right\}$. For with this partition $E$, the relation $\zeta$ clearly reduces to the relation

$$
\zeta=\left\{(a, b) \in S \times S: a^{-1} a=a^{-1} b b^{-1} a \text { and } b^{-1} b=b^{-1} a a^{-1} b\right\} .
$$

If $(a, b) \in \zeta$, then

$$
\begin{aligned}
a a^{-1} & =a\left(a^{-1} a\right) a^{-1}=a\left(a^{-1} b b^{-1} a\right) a^{-1}=\left(a a^{-1}\right)\left(b b^{-1}\right)\left(a a^{-1}\right) \\
& =\left(b b^{-1}\right)\left(a a^{-1}\right)\left(b b^{-1}\right)=b\left(b^{-1} a a^{-1} b\right) b^{-1}=b\left(b^{-1} b\right) b^{-1}=b b^{-1},
\end{aligned}
$$


so $(a, b) \in R$, i.e., $\zeta \subseteq R$. Since $R$ is an idempotent-separating left congruence on $S, R=\zeta$ as required. One may of course provide a direct proof of this result without using Theorem 3.1.

Thus the study of idempotent-separating left congruences on $S$ reduces to the study of those left congruences on $S$ which are contained in $R$. Recall that $T$ is called a full inverse subsemigroup of $S$ if $T$ is an inverse subsemigroup of $S$ which contains all idempotents of $S$.

THEOREM 4.2. The lattice of idempotent-separating left conguences on an inverse semigroup $S$ is isomorphic to the lattice of full inverse subsemigroups of $S$.

Proof. Let $\rho$ be an idempotent-separating left congruence on $S$ with kernel $\operatorname{Ker} \rho=\left\{A(e): e \in E_{S}\right\}$. As usual, set $A=\bigcup_{e \in E_{S}} A(e)$. Then $A$ is a subsemigroup of $S$ which contains $E_{S}$. In addition, if $a \in A$, then $a \in$ $A\left(a a^{-1}\right)$ because $\rho \subseteq R$, and so $a^{-1}=a^{-1}\left(a a^{-1}\right) \rho a^{-1} a \in E_{S}$, i.e., $a^{-1} \in A$. Hence $A$ is a full inverse subsemigroup of $S$.

Conversely, if $A$ is a full inverse subsemigroup of $S$, let $A=\left\{A \cap R_{e}\right.$ : $e \in E_{S}$ \}. We claim that $A$ is a left kernel system of $S$. A clearly satisfies $\mathrm{Ll}$ and L2. If $a \in A$, then $\forall e \in E_{S}, a A(e) \subseteq A$ since $A$ is a subsemigroup of $S$, and also $a A(e) \subseteq a R_{e} \subseteq R_{a e}$ since $R$ is a left congruence on $S$. Hence $a A(e) \subseteq$ $R_{a e} \cap A=A(a e)$, so $\mathrm{L} 3$ is satisfied. Furthermore, $a^{-1} \in A$ so $a^{-1} A(a) \subseteq$ $A\left(a^{-1} a\right)$ by $\mathrm{L} 3$ so $\mathrm{L} 4$ is satisfied. Finally, if $a^{-1} b \in A\left(a^{-1} a\right)$ for some $b \in A$, then $a^{-1} b R a^{-1} a$, so $a^{-1} b b^{-1} a=a^{-1} a$ : Hence $a=a\left(a^{-1} a\right)=$ $a a^{-1} b b^{-1} a=\left(a a^{-1}\right) b\left(b^{-1} a\right) \in A$ (since $a a^{-1}, b, b^{-1} a \in A$ ), and so L5 is satisfied. One can easily check (by using Theorem 2.1) that this establishes an isomorphism between the lattice of idempotent-separating left congruences on $S$ and the lattice of full inverse subsemigroups of $S$.

LEMMA 4.1. If $\rho$ is an idempotent-separating left congruence on $S$ then $\rho_{-1}$ is an idempotent-separating right congruence on $S$ and $\rho \circ \rho_{-1}=\rho_{-1}^{\circ} \rho$ is the smallest equivalence relation containing both $\rho$ and $\rho_{-1}$.

Proof. That $\rho_{-1}$ is an idempotent-separating right congruence on $S$ is obvious. The rest of the proof follows from Lemma 1.4 of [1] and Lallement's result [6] that any left congruence on a regular semigroup which is contained in Green's relation $R$ commutes with any right congruence which is contained in $L$.

The following theorem describes how a $D$-class of $S$ is further partitioned by an idempotent-separating left congruence on $S$. It may be thought of as a generalization of Green's lemma [1, Lemma 2.2] and provides an analogue of Lagrange's theorem for finite groups in the case where $S$ is finite. 
TheOREM 4.3 (A GREen-LAgRANGe THEOREM). Let $\rho$ be an idempotentseparating left congruence on $S$ with kernel $A=\left\{A(e): e \in E_{S}\right\}$. For each $a \in S$ define

$$
\begin{aligned}
& R_{\rho}(a)=\bigcup\left\{H_{x}: x \in a \rho\right\} \\
& L_{\rho}(a)=\bigcup\left\{H_{x}: x \in a \rho_{-1}\right\} \\
& D_{\rho}(a)=\bigcup\left\{H_{x}: x \in a\left(\rho \circ \rho_{-1}\right)\right\}
\end{aligned}
$$

Then

(1) $\forall a \in S, a \rho=a A\left(a^{-1} a\right)$.

(2) If $a L b$ then for some $x, y \in S, x a=b$ and $y b=a$ : the mappings $\phi: u \rightarrow x u \forall u \in a \rho$ and $\psi: v \rightarrow y v \forall v \in b \rho$ are mutually inverse one-

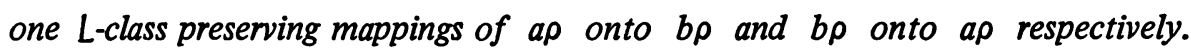

(3) For each $e \in E_{S}, e\left(\rho \cap \rho_{-1}\right)=e \rho \cap H_{e}=e \rho_{-1} \cap H_{e}$ is a subgroup of $H_{e}$ : If $e, f \in E_{S}$ and $f \in D_{\rho}(e)$ then $e\left(\rho \cap \rho_{-1}\right) \cong f\left(\rho \cap \rho_{-1}\right)$.

(4) If $S$ is finite, then for each $a \in S$,

(i) $|b \rho|=|a \rho|$ and $\left|b \rho_{-1}\right|=\left|a \rho_{-1}\right| \forall b \in D_{\rho}(a) ; \quad\left|a \rho \cap H_{a}\right|=\mid b \rho \cap$ $H_{b} \mid$ and $\left|a \rho_{-1} \cap H_{a}\right|=\left|b \rho_{-1} \cap H_{b}\right| \forall b \in D_{\rho}(a)$;

(ii) $|a \rho||| R_{\rho}(a)||\left|D_{\rho}(a)\right|$ and $\left|a \rho_{-1}\right||| L_{\rho}(a)||\left|D_{\rho}(a)\right|$;

(iii) $|a \rho|,\left|a \rho_{-1}\right||| a\left(\rho \circ \rho_{-1}\right) \mid$;

(iv) $|a \rho|=\left|a \rho_{-1}\right|$ if $D_{\rho}(a) \cap E_{S} \neq \square$.

Proof. (1) Let $a x \in a A\left(a^{-1} a\right)$. Then $a x \rho a\left(a^{-1} a\right)=a$, so $a x \in a \rho$. Conversely if $x \rho a$ then $x x^{-1}=a a^{-1}$ and so $x=\left(x x^{-1}\right) x=a a^{-1} x=$ $a\left(a^{-1} x\right)$, and $a^{-1} x \rho a^{-1} a$, so $a^{-1} x \in A\left(a^{-1} a\right)$. Hence $x \in A\left(a^{-1} a\right)$.

(2) This is straightforward and is omitted.

(3) Let $e \in E_{S}$ : Then if $a \in e \rho \cap H_{e},(a, e) \in \rho$ and so $a^{-1}=$ $a^{-1} e \rho a^{-1} a=e$, i.e., $a^{-1} \in e \rho \cap H_{e}$, and so $a \in e \rho_{-1} \cap H_{e}$. It follows easily that $e \rho \cap H_{e}=e \rho_{-1} \cap H_{e}=e\left(\rho \cap \rho_{-1}\right)$. Clearly $e\left(\rho \cap \rho_{-1}\right)$ is a subgroup of $H_{e}$ since $\rho$ induces a left congruence on the group $H_{e}$.

Suppose now that $e, f \in E_{S}$ and that $e \in D_{\rho}(f)$. There are elements $a, b \in S$ such that $b \in H_{f}, e \rho_{-1} a$ and $a \rho b$, and it follows that $a a^{-1}=f$, $a^{-1} a=e, b b^{-1}=b^{-1} b=f, e \rho a^{-1}$ and $a^{-1} \rho_{-1} b^{-1}$. It is straightforward to check that the mapping $\chi: e\left(\rho \cap \rho_{-1}\right) \rightarrow f\left(\rho \cap \rho_{-1}\right)$ defined by $\chi(u)=$ $b^{-1} a u a^{-1} b \forall u \in e\left(\rho \cap \rho_{-1}\right)$ is an isomorphism from $e\left(\rho \cap \rho_{-1}\right)$ onto $f\left(\rho \cap \rho_{-1}\right)$.

(4) Suppose now that $S$ is finite.

(i) First note that by part (2) of the theorem, if $a L b$ then $|a \rho|=|b \rho|$ and $\left|a \rho \cap H_{a}\right|=\left|b \rho \cap H_{b}\right|$. Let $\left|a \rho \cap H_{a}\right|=k$ : Since $H_{a}=\bigcup_{x \in H_{a}}\left(x \rho \cap H_{x}\right)$ 
it follows that $\left|H_{a}\right|=l k$ for some $l \in Z^{+}$. We claim that if $c \in a \rho$ then $\left|a \rho \cap H_{a}\right|=\left|c \rho \cap H_{c}\right|=k$. Let $A=\left\{x \rho \cap H_{a}: x \in H_{a}\right\}$ and $B=\left\{x \rho \cap H_{c}\right.$ : $\left.x \in H_{c}\right\}$ and define $\nu: A \rightarrow B$ by $\nu\left(x \rho \cap H_{a}\right)=x \rho \cap H_{c} \forall x \in H_{a}$. One checks that $\nu$ is well defined and is a one-one mapping of $A$ onto $B$, and so $|A|=|B|$. But $\left|H_{a}\right|=|A|\left|a \rho \cap H_{a}\right|$ and $\left|H_{c}\right|=|B|\left|c \rho \cap H_{c}\right|$, and since $\left|H_{a}\right|=\left|H_{c}\right|$ it follows that $\left|a \rho \cap H_{a}\right|=\left|c \rho \cap H_{c}\right|$. The remainder of (i) follows easily.

(ii) We have $k=\left|b \rho \cap H_{b}\right| \forall b \in D_{\rho}(a)$ and $\left|H_{b}\right|=l k$. Let $n$ be the number of $L$-classes which $a \rho$ intersects and let $m$ be the number of $R$-classes which $a \rho_{-1}$ intersects. Then since $R_{\rho}(a)=\bigcup_{x \in a \rho} H_{x},\left|R_{\rho}(a)\right|=n l k,|a \rho|=$ $n k$ and also $D_{\rho}(a)=\bigcup_{x \in a \rho_{-1}} R_{\rho}(a)$ so $\left|D_{\rho}(a)\right|=m n l k$. Thus $|a \rho||| R_{\rho}(a)||$ $\left|D_{\rho}(a)\right|$ and similarly $\left|a \rho_{-1}\right||| L_{p}(a)||\left|D_{\rho}(a)\right|$.

(iii) We need only note that $a\left(\rho \circ \rho_{-1}\right)=\bigcup_{x \in a \rho_{-1}} x \rho=\bigcup_{x \in a \rho} x \rho_{-1}$, and so $|a \rho|,\left|a \rho_{-1}\right||| a\left(\rho \circ \rho_{-1}\right) \mid$.

(iv) Note that if $e \in E_{S}$ then the mapping $a \rightarrow a^{-1} \forall a \in e \rho$ is a bijection between $e \rho$ and $e \rho_{-1}$ : The result follows easily.

5. Inverse semigroups with only trivial full inverse subsemigroups. We now turn to the question of characterizing inverse semigroups $S$ which have no idempotent-separating left congruences between $i_{S}$ and $R$. In view of Theorem 4.2 this is equivalent to the problem of characterizing those inverse semigroups $S$ which have no full inverse subsemigroups other than $E_{S}$ and $S$. We shall refer to $E_{S}$ and $S$ as the trivial full inverse subsemigroups of $S$.

We use the notation $\langle W\rangle$ to denote the subsemigroup generated by a subset $W$ of $S$. It is clear that if $S$ is an inverse semigroup with only trivial full inverse subsemigroups, then either $S=E_{S}$ is a semilattice or $S=\left\langle a, a^{-1}, E_{S}\right\rangle$ $\forall a \in S \backslash E_{S}$. This comment will be used without further reference.

LEMmA 5.1. If $S=\left\langle a, a^{-1}, E_{S}\right\rangle$ for some $a \in S \backslash E_{S}$ then $S=E_{S} \cup$ $E_{S}\left\langle a, a^{-1}\right\rangle$.

Proof. Let $s$ be an element of $S \backslash E_{S}$. Then

$$
\begin{aligned}
s & =e_{1} w_{1} e_{2} w_{2} \cdots e_{n} w_{n} e_{n+1}, \text { some } e_{i} \in E_{S}, w_{i} \in\left\langle a, a^{-1}\right\rangle, \\
& =e_{1} w_{1} e_{2} w_{2} \cdots e_{n-1} w_{n-1} e_{n} w_{n}\left(w_{n}^{-1} w_{n}\right) e_{n+1} \\
& =e_{1} w_{1} e_{2} w_{2} \cdots e_{n-1} w_{n-1} e_{n}\left(w_{n} e_{n+1} w_{n}^{-1}\right) w_{n} \\
& =e_{1} w_{1} e_{2} w_{2} \cdots e_{n-1} w_{n-1} f_{n} w_{n} \text { where } f_{n}=e_{n} w_{n} e_{n+1} w_{n}^{-1} \in E_{S} .
\end{aligned}
$$

If we proceed inductively in this manner it is easy to see that $s=f_{1} w_{1} \cdots w_{n}$, some $f_{1} \in E_{S}$, and so $s \in E_{S}\left\langle a, a^{-1}\right\rangle$. 
Lemma 5.2. If $S=E_{S} \cup E_{S} A$ for some inverse subsemigroup $A$ of $S$ and if $S$ has only trivial full inverse subsemigroups, then $A$ has only trivial full inverse subsemigroups.

Proof. Suppose that $B$ is a proper full inverse subsemigroup of $A$ and set $B_{1}=E_{S} \cup E_{S} B$. A standard argument shows that $B_{1}$ is a full inverse subsemigroup of $S$. Furthermore, since $B \subseteq E_{S} B \subseteq B_{1}$ and since $B$ contains nonidempotents, $B_{1} \neq E_{S}$ and so $B_{1}=S$. Now let $x$ be any element of $A$ : Then $x \in S$ and so $x \in B_{1}$. If $x \in E_{S}, x \in A \cap E_{S} \subseteq B$ and so $x \in B$. If $x \notin E_{S}$, then $x=f b$ for some $f \in E_{S}, b \in B$. Thus $x x^{-1}=f b b^{-1} \in E_{S} \cap A \subseteq B$, and so $x=f b=\left(f b b^{-1}\right) b \in B$. This yields $A \subseteq B$ and so $A=B$ which contradicts the fact that $B$ is proper. Hence $A$ has no proper full inverse subsemigroups.

We recall that an inverse semigroup $S$ is called an elementary inverse semigroup if $S$ is generated by an element and its inverse, i.e., $S=\left\langle a, a^{-1}\right\rangle$ for some $a \in S$. We make use of the following lemma concerning elementary inverse semigroups due to L. M. Gluskin [3] .

LEMMA 5.3 (GLuSKIN [2]). If $S$ is an elementary inverse semigroup generated by $a$ and $b\left(=a^{-1}\right)$ then every element $w \in S$ is expressible in the form

$$
w=a^{i} b^{j} a^{k}, \quad 0 \leqslant i, k \leqslant j, \quad j \geqslant 1 .
$$

Furthermore, if $l \geqslant i$ and $n \geqslant k$, then $a^{l} b^{m} a^{n}=a^{i} b^{j} a^{k}$ implies that $b^{m}=$ $b^{j+(n-k)+(l-i)} ;$ if $l \leqslant i$ and $n \geqslant k$, then $a^{l} b^{m} a^{n}=a^{i} b^{j} a^{k}$ implies that $b^{m+(i-l)}=b^{j+(n-k)}$.

We deduce a number of technical corollaries and lemmas concerning elementary inverse semigroups. The arguments involved in the first two of these are quite routine, involving only the commuting of idempotents, the expansion $x=x x^{-1} x$ and Lemma 5.3, so we omit these details and provide statements of the results without proof.

COROLlaRY 5.1. If $S$ is an elementary inverse semigroup generated by $a$ and $b\left(=a^{-1}\right)$ and if $a$ is of infinite order then

(a) $a^{l} b^{m} a^{n}=a^{i} b^{j} a^{k}$ implies $m-l-n=j-i-k$;

(b) $a^{i} b^{j} a^{k} \in E_{S}$ iff $i+k=j$.

LEMMA 5.4. If $S$ is an elementary inverse semigroup generated by $a$ and $b\left(=a^{-1}\right)$ then multiplication in $S$ is defined by 


$$
\left(a^{i} b^{j} a^{k}\right)\left(a^{l} b^{m} a^{n}\right)= \begin{cases}a^{i} b^{j+m-k-l} a^{n} & \text { if } k+l \leqslant j, m, \\ a^{i} b^{j} a^{k+l-m+n} & \text { if } m \leqslant k+l \leqslant j, \\ a^{k+l-j+i} b^{m} a^{n} & \text { if } j \leqslant k+l \leqslant m, \\ a^{k+l-j+i} b^{k+l} a^{k+l-m+n} & \text { if } k+l \geqslant j, m .\end{cases}
$$

From these two results we obtain the following

COROLLARY 5.2. If $S$ is an elementary inverse semigroup generated by $a$ and $b$ and if $S$ has only trivial full inverse subsemigroups, then $a$ is of finite order.

Proof. Suppose that $a$ is of infinite order. As in Lemma 5.1, $S=$ $\left\langle E_{S}, a^{2}, b^{2}\right\rangle$ and thus $a \in\left\langle E_{S}, a^{2}, b^{2}\right\rangle$. Hence $a=e w$ for some $e \in E_{S}$, $w \in\left\langle a^{2}, b^{2}\right\rangle$ and by the preceding lemmas, $e=a^{i} b^{i+j} a^{j}$ and $w=a^{2 k} b^{2 l} a^{2 m}$ for some integers $i, j, k, l$ and $m$ such that $i, j \geqslant 0,0 \leqslant k, m \leqslant l$. Thus by the previous lemma

$$
a= \begin{cases}a^{i} b^{i+j+2 l-j-k} a^{2 m} & \text { if } j+2 k \leqslant i+j, 2 l, \\ a^{i} b^{i+j} a^{j+2 k-2 l+2 m} & \text { if } 2 l \leqslant j+2 k \leqslant i+j, \\ a^{j+2 k-i-j+i} b^{2 l} a^{2 m} & \text { if } i+j \leqslant j+2 k \leqslant 2 l, \\ a^{j+2 k-i-j+i} b^{j+2 k} a^{j+2 k-2 l+2 m} & \text { if } j+2 k \geqslant i+j, 2 l .\end{cases}
$$

Then since $a$ is of infinite order, Corollary 5.1 yields $2 l-2 k-2 m=-1$, which is a contradiction since $2 l-2 k-2 m$ is even. Hence $a$ is of finite order.

LEMMA 5.5. If $S$ is an elementary inverse semigroup with only trivial full inverse subsemigroups, then either $S$ is a group of prime order or $S$ is the five-element Brandt semigroup.

Proof. Let $S$ be generated by $a$ and $b\left(=a^{-1}\right)$. By Corollary 5.2, $\exists m, n \in Z^{+}$such that $m>n$ and $a^{m}=a^{n}$. With $m$ and $n$ chosen minimally it follows easily (using Gluskin's Lemma 5.3) that $G=\left\{a^{n}, a^{n+1}, \cdots, a^{m-1}\right\}$ $=\left\{b^{n}, \cdots, b^{m-1}\right\}$ is a cyclic group and an ideal of $S$.

Now set $A=E_{S} \cup G$ : since $G$ is an ideal of $S, A$ is a full inverse subsemigroup of $S$ and hence $A=S$ or $A=E_{S}$. If $A=S$, then $a \in E_{S}$ or $a \in G$, and if $a \in E_{S}$ then $a=a^{-1}$ and $S=\{a\}:$ If $a \in G$ then $S=G$ is a group which has no proper subgroups and so is a cyclic group of prime order.

Suppose now that $A=E_{S}$. Then $G=\{0\}$ because $G$ is an ideal of $S$ and is a group consisting of idempotents: We claim now that this implies that 
$a^{2}=b^{2}=0$. Suppose that $a^{2} \neq 0$ : Then $a^{n}=0$ for some $n>2$ and $\left\{a, a^{2}, \cdots, a^{n-1}, a^{n}=0\right\}$ and $\left\{b, b^{2}, \cdots, b^{n-1}, b^{n}=0\right\}$ are sets of $n$ distinct elements with $a^{i} \notin E_{S}$ and $b^{i} \notin E_{S}$ for $i<n$. Now set $B=E_{S} \cup$ $E_{S}\left\langle a^{2}, b^{2}\right\rangle . B$ is clearly a full inverse subsemigroup of $S$ and $B \neq E_{S}$ since $a^{2} \in B$; we produce a contradiction by showing that $a \notin B$, and so $B \neq S$. First note that if $e$ is an idempotent of $S$ other than 0 then $e=a^{i} b^{i+j} a^{j}$ for some $i, j \in Z^{+} \cup\{0\}$ such that $0<i+j<n$. (This follows by a routine argument using the fact that the elements $b, b^{2}, \cdots, b^{n-1}$ are all distinct.) That $a \notin E_{S}\left\langle a^{2}, b^{2}\right\rangle$ may be proved by an argument along the same lines as the argument used in Corollary 5.2. Thus $a^{2}=b^{2}=0$ and so $S=\{a, b, a b, b a, 0\}$. One checks easily that the elements $a, b, a b$ and $b a$ are all distinct, and thus $S$ is the five-element Brandt semigroup (with nonzero idempotents $a b$ and $b a$ ).

LEMma 5.6. Let $S=\left\langle E_{S}, a, a^{-1}\right\rangle$ be an inverse semigroup with only trivial full inverse subsemigroups and suppose that $\left\langle a, a^{-1}\right\rangle=G$ is a cyclic group of prime order with identity $e_{0} \in E_{S}$. Then $S=E_{S} \cup G$ with multiplication between $E_{S}$ and $G$ defined by

$$
\begin{array}{ll}
e g=g e=e e_{0} & \text { if } e \in E_{S}, g \in G \text { and } e e_{0} \neq e_{0}, \\
e g=g e=g & \text { if } e \in E_{S}, g \in G \text { and } e e_{0}=e_{0} .
\end{array}
$$

Proof. From Lemma 5.2 we know that $S=E_{S} \cup E_{S} G$. Before embarking on the proof we recall that the natural partial ordering on $E_{S}$ is defined by $e \leqslant f$ iff $e f=f e=e$ : We make liberal use of this partial ordering in the proof. Note that for each $e, f \in E_{S}$, ef $\leqslant e$ and $e f \leqslant f$.

We first claim that for each $e \in E_{S}, R_{e e_{0}}=\left\{e a^{i}: i=1, \cdots, p\right\}, L_{e e_{0}}=$ $\left\{a^{i} e: i=1, \cdots, p\right\}$, and $D_{e e_{0}}=\left\{a^{i} e a^{j}: i, j=1, \cdots, p\right\}$. Clearly $\left\{e a^{i}\right.$ : $i=1, \cdots, p\} \subseteq R_{e e_{0}}$. Suppose now that $s \in R_{e e_{0}}$ : If $s \in E_{S}$ then $s=e e_{0}$; if $s \notin E_{S}$ then $s=f a^{i}$, some $f \in E_{S}, i \in\{1, \cdots, p\}$, and so $e e_{0}=s s^{-1}=$ $f a^{i} a^{-i} f=f e_{0} f=f e_{0}$, so $s=f a^{i}=f e_{0} a^{i}=e e_{0} a^{i}=e a^{i}$. A similar argument shows that $L_{e e_{0}}=\left\{a^{i} e: i=1, \cdots, p\right\}$. It follows that the set of idempotents of $D_{e e_{0}}$ is $\left\{a^{i} e a^{-1}: i=1, \cdots, p\right\}$, and if $x R a^{i} e a^{-i}$ for some $i$ then $x=$ $a^{i} e a^{-1} a^{j}=a^{i} e a^{k}$, some $k$. Thus $D_{e e_{0}}=\left\{a^{i} e a^{j}: i, j=1, \cdots, p\right\}$.

We now claim that in fact $D_{e e_{0}}=\left\{e e_{0}\right\}$ if $e e_{0} \neq e_{0}$ (i.e., if $e e_{0}<e_{0}$ ). Suppose that $D_{f_{0} e_{0}} \neq\left\{f_{0} e_{0}\right\}$ for some $f_{0} \in E_{S}$ for which $f_{0} e_{0} \neq e_{0}$, and consider $B=\bigcup_{e \leqslant f_{0} e_{0}} D_{e}$ and let $A=E_{S} \cup B$ : We claim that $A$ is a full inverse subsemigroup of $S$. It clearly suffices to show that $A$ is a subsemigroup of $S$ since if $x \in D_{e}$ then $x^{-1} \in D_{e}$. Let $a^{i} e f_{0} e_{0} a^{j}, a^{k} f f_{0} e_{0} a^{l} \in B$. Then

$$
a^{i} e f_{0} e_{0} a^{j} a^{k} f f_{0} e_{0} a^{l}=a^{i+j+k}\left(a^{-j-k} e f_{0} e_{0} a^{j+k} f f_{0} e_{0}\right) a^{l} \in B .
$$


(Note that $a^{-j-k} e f_{0} e_{0} a^{j+k} f f_{0} e_{0} \in E_{S}$ and $a^{-j-k} e f_{0} e_{0} a^{j+k} f f_{0} e_{0} \leqslant f_{0} e_{0}$.) Also, if $f \in E_{S}$ and $a^{i} e f_{0} e_{0} a^{j} \in B$, then $f\left(a^{i} e f_{0} e_{0} a^{j}\right)=a^{i}\left(a^{-i} f a^{i}\right) e f_{0} e_{0} a^{j} \in B$ since $a^{-i} f a^{i} e f_{0} e_{0} \leqslant f_{0} e_{0}$; similarly $\left(a^{i} e f_{0} e_{0} a^{j}\right) f \in B$, and thus $A$ is a full inverse subsemigroup of $S$. Now $D_{f_{0} e_{0}} \neq\left\{f_{0} e_{0}\right\}$ and $D_{f_{0} e_{0}} \subseteq A$, so $A \neq E_{S}$. On the other hand $a \in D_{e_{0}}$ and $e_{0} \leqslant e_{0} f_{0}$, so $a \notin A$, so $A \neq S$. This contradicts the fact that $S$ has no full inverse subsemigroups other than $E_{S}$ and $S$. Hence $D_{e e_{0}}=\left\{e e_{0}\right\}$ if $e e_{0} \neq e_{0}$. Thus $a^{i} e e_{0}=a^{i} e=e e_{0} a^{i}=e a^{i}=e e_{0} \forall i=1, \cdots, p$ provided $e e_{0} \neq e_{0}$. Clearly, if $e e_{0}=e_{0}$, then $a^{i} e=a^{i} e_{0} e=a^{i} e_{0}=a^{i}=e a^{i}$. This proves the lemma.

Lemma 5.7. Let $S=\left\langle E_{S}, a, a^{-1}\right\rangle$ be an inverse semigroup with only trivial full inverse subsemigroups and suppose that $\left\langle a, a^{-1}\right\rangle=T$ is the fiveelement Brandt semigroup $T=\left\{a, b, e_{1}, e_{2}, e_{0}\right\}$ (where $b=a^{-1}, e_{1}=a a^{-1}$, $e_{2}=a^{-1} a, e_{0}$ is the zero). Then $S=E_{S} \cup T$ with multiplication between $E_{S}$ and $T$ defined by

$(* *)$

$$
\begin{aligned}
& e a=e e_{1} \text { if } e e_{1} \neq e_{1}, \\
& =a \quad \text { if } e e_{1}=e_{1} \text {; } \\
& a e=e e_{2} \text { if } e e_{2} \neq e_{2} \text {, } \\
& =a \text { if } e e_{2}=e_{2} \text {; } \\
& e b=e e_{2} \quad \text { if } e e_{2} \neq e_{2}, \quad b e=e e_{1} \text { if } e e_{1} \neq e_{1} \text {, } \\
& =b \quad \text { if } e e_{2}=e_{2} ; \quad=b \quad \text { if } e e_{1}=e_{1} \text {. }
\end{aligned}
$$

Proof. This is similar in spirit to the proof of Lemma 5.6 so we provide only an outline of the proof. One checks first that for each $e \in E_{S}, R_{e e_{1}}=$ $\left\{e e_{1}, e a\right\}, R_{e e_{2}}=\left\{e e_{2}, e b\right\}, L_{e e_{1}}=\left\{b e, e e_{1}\right\}, L_{e e_{2}}=\left\{e e_{2}, a e\right\}$ and so $D_{e e_{1}}=\left\{e e_{1}, e a, b e, b e a\right\}$ and $D_{e e_{2}}=\left\{e e_{2}, e b, a e, a e b\right\}$. Since $a^{2}=b^{2}=e_{0}$ and since $S=E_{S} \cup E_{S} T$, it follows that $S=E_{S} \cup\left(\cup_{e \leqslant e_{1}} D_{e}\right) \cup\left(\cup_{e<e_{2}} D_{e}\right)$. One then proves, as in the proof of Lemma 5.6, that $D_{e e_{1}}=\left\{e e_{1}\right\}$ if $e e_{1} \neq e_{1}$ and that $D_{e e_{2}}=\left\{e e_{2}\right\}$ if $e e_{2} \neq e_{2}$. The result $S=E_{S} \cup T$ and the multiplication (**) then follow easily.

Motivated by the results of Lemmas 5.6 and 5.7 we introduce two semigroups $F\left(E, e_{0}, G\right)$ and $F\left(E, e_{0}, e_{1}, e_{2}, a, b\right)$ as follows:

(1) Let $E$ be a semilattice, $e_{0}$ a fixed element of $E$ and $G$ a group with identity $e_{0}$ : We define $F\left(E_{1}, e_{0}, G\right)=E \cup G$ with multiplication inherited by that in $E$ and $G$ and multiplication between elements of $E$ and elements of $G$ defined by (*) (in Lemma 5.6).

(2) Let $E$ be a semilattice, $e_{0}, e_{1}$ and $e_{2}$ distinct fixed elements of $E$ for which $e_{0} \leqslant e_{1}, e_{0} \leqslant e_{2}$ and let $a, b$ be elements not in $E$ : let $T=$ $\left\{a, b, e_{0}, e_{1}, e_{2}\right\}$ be the five-element Brandt semigroup with $b=a^{-1}, e_{1}=a b$, $e_{2}=b a$ and $e_{0}$ as zero. Now define $F\left(E, e_{0}, e_{1}, e_{2}, a, b\right)=E \cup T$ with 
multiplication inherited by that in $E$ and $T$ and multiplication between elements of $E$ and elements of $T$ defined by (**) (in Lemma 5.7).

Combining all of the results of this section we have the following theorem.

THEOREM 5.1. An inverse semigroup $S$ has no proper full inverse subsemigroups (other than $E_{S}$ and $S$ ) iff either

(i) $S \cong F\left(E, e_{0}, e_{1}, e_{2}, a, b\right)$ for some semilattice $E$ and elements $e_{0}$, $e_{1}, e_{2} \in E$ and $a, b \notin E$; or

(ii) $S \cong F\left(E, e_{0}, G\right)$ for some semilattice $E$, some $e_{0} \in E$ and some cyclic group $G$ of prime order.

REMARKS. (1) Semilattices and cyclic groups of prime order are special cases of the semigroup $F\left(E, e_{0}, G\right)$.

(2) Theorem 5.1 also characterizes inverse semigroups $S$ which have no idempotent-separating left (right) congruences between $i_{S}$ and $R(L)$.

(3) Theorem 5.1 suggests that a general study of the connection between the lattice of full inverse subsemigroups of an inverse semigroup and the structure of the semigroup might prove rewarding.

I should like to offer my thanks to Professor G. B. Preston, who originally suggested the problem solved in Theorem 2.1. The results of this paper were presented at a symposium on inverse semigroups and their generalizations at the University of Northern Illinois in February, 1973.

\section{REFERENCES}

1. A. H. Clifford and G. B. Preston, The algebraic theory of semigroups. Vols. I, II, Math. Surveys, no. 7, Amer. Math. Soc., Providence, R. I., 1961, 1967. MR 24 \#A2627; 36 \#1558.

2. L. M. Gluskin, Elementary generalized groups, Mat. Sb. 41 (83) (1957), 23-36. (Russian) MR 19, 836.

3. J. M. Howie, The maximum idempotent-separating congruence on an inverse semigroup, Proc. Edinburgh Math. Soc. (2) 14 (1964/65), 71-79. MR 29 \#1275. MR 16, 215.

4. G. B. Preston, Inverse semigroups, J. London Math. Soc. 29 (1954), 396-403.

5. N. R. Reilly and H. E. Scheiblich, Congruences on regular semigroups, Pacific J. Math. 23 (1967), 349-360. MR 36 \#2725.

6. G. Lallement, Demi-groupes réguliers, Ann. Mat. Pura Appl. (4) 77 (1967), 47-129. MR 37 \#1505.

DEPARTMENT OF MATHEMATICS, UNIVERSITY OF NEBRASKA, LINCOLN, NEBRASKA 68508 